\title{
Etiopathogenesis of Melasma
}

\section{Fabrizio Guarneri*}

Department of Clinical and Experimental Medicine - Dermatology, University of Messina, Messina, Italy

\begin{abstract}
Known since ancient times, melasma is notoriously a frequent dyspigmentation disorder characterized by localized, chronic acquired cutaneous hypermelanosis. Despite high frequency, significant negative effects on quality of life and often unsatisfactory results of available treatment, not much research has been performed on the etiopathogenesis of this disease, which still remains largely obscure. An extensive review of the literature on etiology and pathogenesis of melasma is presented here, in which known causal/exacerbating factors (sun exposure, alterations of hormonal balance, use of cosmetics and photosensitizing drugs, procedures and inflammatory processes of the skin, stressful events) are linked with their effects on multiple metabolic pathways of melanocytes and other components of the socalled "melanin unit". Available data outline an incompletely defined, but certainly complex and somehow fascinating network of interactions of structural and functional alterations, which deserves further investigation, not only to obtain new and better treatments for melasma, but also -and probably more important in view of a more detailed understanding of the entire physiology and pathophysiology of melanogenesis, with many possible applications in the vast field of dyspigmentation disorders.
\end{abstract}

Keywords: Melasma; Melanogenesis; Melanin unit; Etiopathogenesis; Pathogenic mechanisms; Inflammation; Hormones; Genetics

Abbreviations: BC: Before Christ; UV: Ultraviolet; NCBI: National Center For Biotechnology Information; Mirna: Microrna; IL-1: Interleukin 1; MSH: Melanocyte Stimulating Hormone; ACTH: Adrenocorticotropic Hormone; LH: Luteinizing Hormone; FSH: Follicle-Stimulating Hormone; MC1R: Melanocortin Type 1 Receptor; ER: Estrogen Receptor ; PR :Progesterone Receptor; TPO: Thyroid Peroxidase; TSH: Thyroid Stimulating Hormone; Inos: Inducible Nitric Oxide Synthase ; VEGF: Vascular Endothelial Growth Factor ; GM-CSF: Granulocyte-Macrophage Colony Stimulating Factor; Mrna: Messenger RNA; NF: Nuclear Factor; NGFR: Nerve Growth Factor Receptor; TYRP1: Tyrosinase-Related Protein 1

\section{Introduction}

Clinically described -according to available documents- already at the times of Hippocrates (470-360 BC), but fully characterized, classified and extensively studied only in the last 80 years, melasma notoriously is a dysfunction of melanogenesis which results in localized, chronic acquired cutaneous hypermelanosis [1]. It is one of the most common pigmentary disorders observed by dermatologists: as an example, some studies give an estimate of five million people affected in the United States of America alone [2]. While it can occur in all ethnic and population groups, frequency is higher in women in menacme with Fitzpatrick's skin phototype III, IV or V, living in areas of high ultraviolet (UV) light exposure [3]. Improvements are usually observed in winter and worsening in summer or after intense photoexposure [1]. Melasma has a significantly negative impact on the quality of life of patients, causing shame, low self-esteem, anhedonia, dissatisfaction and, in some cases, suicidal ideation. Another important issue is the direct and indirect cost of treatments, which is often high and not rewarded by results satisfactory for the patient [1]

For the above reasons, melasma represents a topic of interest for medical researchers: a search in PubMed for the name of the disease, performed on October 4, 2014, yields 7766 articles. However, efforts appear to be mainly focused on treatment, while some aspects of this disease still remain unclear, particularly for which concerns its etiopathogenic mechanisms. This review aims to critically review and evaluate the available literature, and present a picture, as complete as possible, of the "state of the art" of research on the etiopathogenesis of melasma.

\section{Methods}

A search was performed in the PubMed database of the NCBI (National Center for Biotechnology Information), using the string "melasma AND pathogen*". Articles matching this query were then retrieved and manually reviewed, to select only those containing information about the etiopathogenesis of melasma. Further articles on specific aspects or mechanisms were found in the reference list of the selected papers.

\section{Results and Discussion}

As of October 04, 2014, the NCBI PubMed database contained 297 articles matching the query "melasma AND pathogen". Manual review of these papers allowed us to identify 18 articles pertaining to the etiopathogenesis of melasma.

In a 2011 review [3], Sheth and Pandya declared that "the exact underlying etiology for melasma remains a mystery": this view is essentially confirmed in the most recent paper on the topic, published in 2014 [1]. However, although the complete picture is still unclear, many details keep emerging from clinical and laboratory research, and outline a complex disease, resulting from the interaction of several genetic factors, inherited with non-Mendelian patterns, and multiple environmental triggering factors, which include not only sun exposure, but also paraphysiological/pathological/iatrogenic alterations of hormonal balance (due to pregnancy, use of oral contraceptives and other steroids, hormone replacement therapy, ovarian tumors), use of cosmetics and photosensitizing drugs, procedures and inflammatory processes of the skin, stressful events [1,4-7]. Additionally, most recent researches on altered expression of several genes (more than 270) as well as metabolic and even structural modifications in melasma show that multiple pathways are involved, related not only to melanogenesis, but

*Corresponding author: Guarneri F, Viale Annunziata, Residence dei Fiori, villa 798168 Messina, Italy,Tel: +39 090 357070; Fax: +39 090 2927691; E-mail: f.guarneri@tiscali.it

Received: October 20, 2014; Accepted: October 22, 2014; Published: October 24, 2014

Citation: Guarneri F (2014) Etiopathogenesis of Melasma. Pigmentary Disorders S1:003. doi: 10.4172/2376-0427.S1-003

Copyright: ( 2014 Guarneri F. The terms of the Creative Commons Attribution License, which permits unrestricted use, distribution, and reproduction in any medium, provided the original author and source are credited. 
also to inflammatory response, oxidative stress and lipid metabolism. There is increasing evidence that melanocytes are only a part of a complex network of communicating cells, and melasma results from alterations of this "melanin unit" rather than of melanocytes or any other cell type alone.

\section{Genetic factors}

Skin phototype III, IV or V and female gender are the most known genetic predisposing factors for melasma. However, several studies suggest the possible role of other inheritable characters, probably multigenic. The biggest research in this field was performed some years ago in dermatology centers of nine countries (United States of America, France, Germany, Netherlands, Mexico, Italy, Singapore, South Korea, Hong Kong) and showed that $48 \%$ of 324 women with melasma had a positive family history for the same disease, and in $97 \%$ of cases the other family member affected was a first degree relative [8]. Epidemiological data in this regard can significantly differ among populations: percentages of positive family history reported in literature are $70.4 \%$ for male patients and 56\% for female patients in Brazil [4,9], 54.7\% in Iran [10], 33\% in India [11], 10.2\% in Singapore [12]. Although sparse and sometimes not involving large patient samples, these studies, which show remarkable differences even between populations living in similar environmental conditions, suggest that susceptibility to melasma is poligenic and, as recently postulated by some authors, could be also influenced by epigenetic modulation of melanogenesis [13]. In detail, Dynoodt et al. [13], found that the expression of sixteen microRNA (miRNA) was different between melanocytes treated with forskolin plus solar-simulated UV irradiation and untreated melanocytes; one of these miRNAs, namely miR-145, was significantly downregulated and able to influence expression of several major pigmentation genes (Sox9, Mitf, Tyr, Trp1, Myo5a, Rab27a, Fscn1).

\section{Sun exposure}

The most important and demonstrated environmental triggering factor of melasma is exposure to sunlight. Among the different components of sunlight, UV radiations (A and $\mathrm{B}$ ) have a major role: they can induce/increase melanogenesis, melanocyte proliferation and migration directly, but also indirectly, through stimulation of the production of interleukin 1 (IL-1), endothelin 1, alpha-melanocyte stimulating hormone ( $\alpha-\mathrm{MSH})$ and adrenocorticotropic hormone (ACTH) by keratinocytes $[3,14]$.

The role of visible and infrared radiations in melanogenesis is much less significant, but not necessarily negligible: a connection between occupational abundant exposure to heat or intense artificial light and worsening of melasma and/or low response to treatment was reported by some authors $[1,15,16]$. An indirect proof of the role of visible light is contained in a recent paper by Castanedo-Cazares et al. [17], they showed that a sunscreen absorbing ultraviolet radiations and visible light enhances the depigmenting efficacy of hydroquinone more than a sunscreen which blocks ultraviolet radiations only.

\section{Hormonal balance}

Although the mechanism(s) that link hormones and melasma are not yet completely clear, it has been demonstrated that melanocytes, even in healthy skin, possess nuclear and cytosolic estrogen receptors, and the number of such receptors is increased in lesional skin of patients with melasma $[18,19]$. Effects on melanocytes depend on the type of hormone: MSH, ACTH, Luteinizing Hormone (LH) and FollicleStimulating Hormone (FSH) increase melanocyte size and production of tyrosinase, while estradiol, estriol and progesterone slightly increase melanocyte proliferation and do not affect or downregulate tyrosinase activity $[18,20]$. However, studies on patients show results often conflicting among themselves and with in vitro data: Hassan et al. [21], found a significant increase of $17-\beta$-estradiol at the beginning of menstrual cycle, Mahmood et al. [22] found that estradiol levels are increased both in the follicular and luteal phase, Perez et al. [23], found significantly higher levels of $\mathrm{LH}$, lower levels of estradiol and no significant variations, in comparison with controls, of $\beta-\mathrm{MSH}, \mathrm{ACTH}$, $\mathrm{FSH}$, progesterone, prolactin, thyroid hormone or cortisol.

The mechanisms by which these hormones can influence melanogenesis were only partially defined. It is known that estrogens bind to nuclear receptors and exert their action on melanocytes in a nongenomic fashion [1,24]. Demonstrated effects in vitro include increased expression of two proteins involved in the pathogenesis of melasma: melanocortin type 1 receptor (MC1R) [25-27] and the scaffolding protein produced by the PDZK1 gene, which promotes the transcription of tyrosinase without inducing modifications of the number of melanocytes or keratinocytes [28].

Kim et al. focused their attention on the $\mathrm{H} 19$ gene, which produces a non-coding RNA but operates in imprint with the gene of insulinlike growth factor type II, named IGF2. Interestingly, a decrease of H19 transcription induces melanogenesis and transfer of melanin to keratinocytes in co-cultures of melanocytes and keratinocytes, but not in cultures of melanocytes alone. This implies an active role of keratinocytes in melanogenesis (and possibly in its dysfunctions, including melasma) and suggests the possible role of $\mathrm{H} 19$ in the pathogenesis of melasma. Also of interest, co-cultures of melanocytes and keratinocytes without $\mathrm{H} 19$ respond to estrogens with an increased production of tyrosinase, an enzyme notoriously involved in melanogenesis, and this could explain, at least in part, the observed correlation between estrogen levels and melasma [29,37].

Some authors studied also the expression of Estrogen Receptor (ER) and Progesterone Receptor (PR), comparing lesional and nonlesional skin of patients with melasma: PR was significantly more expressed in the epidermis of lesional than of nonlesional skin, while this was not the case in the dermis; ER $\beta$ had, instead, increased dermal expression, particularly around small blood vessels and fibroblast-like cells, and non-significantly raised epidermal expression in lesional areas in comparison to unaffected skin [30].

Pregnancy or use of oral contraceptives is conditions of alteration of hormone balance, which are linked to melasma. In older as well as in recent studies, the percentage of women who developed melasma as a direct result of oral contraceptive use are very similar: in 1967 Resnik reported a frequency of $29 \%$ [31], in 2009 Ortonne et al. reported a frequency of $25 \%$ [8]. Of interest, in the above study by Resnik [31] it was shown than $87 \%$ of the women who developed the disease after oral contraceptive use presented melasma also during pregnancy. During pregnancy, particularly in the third trimester, higher levels of $\mathrm{MSH}$, estrogen and progesterone increase production of tyrosinase and dopachrome tautomerase: this might justify the higher susceptibility to the development of melasma, although a detailed explanation of the onset of the disease is not yet available [8,32].

Few papers (of which almost all are case reports) were published on the correlation between melasma and hormones in men. The biggest study on this topic was made by Sialy et al. [33], on 15 subjects, who had raised levels of LH and decreased levels of testosterone.

Concerning non-sexual hormones, some authors suggested a correlation between melasma and thyroid hormones. Lutfi et al. [34], found that thyroid disorders are four times more frequent in melasma 
patients than in healthy controls, and significant differences were also reported by Perez et al. [23], However, the matter is still controversial: a study by Yazdanfar et al. [35], showed no difference in the levels of anti-thyroid peroxidase antibodies (anti-TPO), T3, T4 and Thyroid Stimulating Hormone (TSH) between melasma patients and controls, and similar results were also reported by Sacre et al. [36].

\section{Other factors}

Among the other causal/triggering factors of melasma, inflammation is one of the most studied. Inflammatory mediators like inducible nitric oxide synthase (iNOS), vascular endothelial growth factor (VEGF), granulocyte-macrophage colony stimulating factor (GM-CSF), c-kit, endothelin-1 and stem cell factor are overexpressed in lesional skin of melasma patients, and induce local increase of vessels and inflammatory cells $[37,38]$.

Concerning nitric oxide, Jo et al. [39] found that iNOS protein and iNOS mRNA are overexpressed in keratinocytes of the basal layer of the epidermis in lesional skin of melasma patients, but not in normal skin. Consistently, they found that phosphorylated Akt co-localized with high levels of iNOS: it is well known that phosphorylation of Akt is important for the activation of the transcription factor nuclear factor (NF)-kappaB, which in turn upregulates iNOS expression. An evaluation of the overall oxidoreductive status in patients with melasma was very recently performed by Seçkin et al. [40], who demonstrated an imbalance between oxidants and anti-oxidants, leading to increased oxidative stress.

Particular interest was focused on stem cell factor: Kang et al. [41] showed that in lesional skin it is overexpressed around dermal fibroblasts (and c-kit is overexpressed in the basal epidermal layer), Grichnik et al. [42] demonstrated that it can increase number, size and dendricity of melanocytes and Costa et al. [43] found that half of the patients receiving subcutaneous injections of stem cell factor for the treatment of breast cancer developed hyperpigmentation at injection sites. Jang et al. [44] found that significant differences exist, in this regard, between male and female melasma: male patients have a significantly higher expression of stem cell factor and c-kit, an increased lesion to nonlesion ratio of stem cell factor and c-kit, increased vascularity and increased lesion to nonlesion ratio of the vessel area.

Even cosmetics [45] or cosmetic treatments such as peelings or lasers can elicit melasma, as suggested by Negishi et al. [46], who highlighted the possible role of treatments with intense pulsate light.

Further support to the idea of a link between inflammation and melasma is given by few studies that show a higher frequency of the disease in subjects with post-inflammatory hyperpigmentation $[47,48]$.

With various mechanisms, ranging from photosensitization to deposition in different cutaneous layers to stimulation of melanogenesis, many substances were linked to melasma: the long list includes anticonvulsivants, antimalarials, tetracyclines, amiodarone, sulfonylureas, iron, copper, gold, silver, arsenic, bismuth. However, the matter is still debated; as such correlation was not confirmed by other authors [49].

Neural and psychological factors were postulated by some authors. Bak et al. [50] compared lesional and nonlesional skin of six Asian women with melasma, finding that expression of nerve growth factor receptor (NGFR), neural endopeptidase and nerve fibers was increased in the superficial dermis of areas affected by the disease. Stressful events and affective disorders were considered as triggers in patients reported by Tamega Ade et al. [4] and Wolf et al. [6], and the pathogenic hypothesis formulated is that $\mathrm{ACTH}$ and $\mathrm{MSH}$, produced as organic response to stress, activate melanocortin receptors located in melanocytes, thus inducing melanogenesis.

Finally, some data suggest a possible etiopathogenic role of alterations of lipid metabolism. A transcriptional profiling performed by Kang et al. [51] showed that 279 genes are differentially expressed in lesional and perilesional skin of melasma patients. Upregulation concerns not only known melanogenesis-associated genes, such as tyrosinase and TYRP1 (tyrosinase-related protein 1), but also of a subset of Wnt signaling modulators, including Wnt inhibitory factor 1 , secreted frizzled-related protein 2, Wnt5a. In the same paper, the authors demonstrated downregulation of the majority of lipid metabolism-associated genes, with consequent impairment of skin barrier function. Similar conclusions about lipid metabolism-associated genes in melasma and their effects on barrier function were obtained by Lee et al. [52], who demonstrated a reduced expression of the PPAR $\alpha$, ALOX15B, DGAT2L3 and PPARGC1A genes.

\section{Structural alterations of skin in melasma}

Histological, histochemical, immunohistochemical and molecular techniques show that skin of melasma patients presents several structural, other than functional, alteration, and this further adds to the complexity of the etiopathogenesis of this disease.

Hernández-Barrera et al. [53], comparing lesional and nonlesional skin, found that cutaneous areas affected by melasma have significantly abundant elastotic material, and in these elastotic areas mast cells are significantly increased and prominent among other inflammatory cells.

Torres-Álvarez et al. [54], in a study on lesional, perilesional and photoprotected nonlesional skin of patients, confirmed the overexpression of stem cell factor and c-kit in lesional dermis and epidermis, respectively, and the prevalence of mast cells in lesional dermis, and also discovered some unexpected damage of the basal membrane. In detail, they observed many c-kit positive and pigmented basal cells protruding into the dermis in $70 \%$ and $29 \%$ of lesional and perilesional skin samples, respectively.

More recently, Brianezi et al. [55] compared nuclear morphological features and chromatin texture between basal keratinocytes in facial melasma and adjacent normal skin, and found that in the first case nuclei show larger size, irregular shape, hyperpigmentation and chromatin heterogeneity by fractal dimension, concluding that "the phenotype differences between melasma and adjacent facial skin can result from complete epidermal melanin unit alterations, not just hypertrophic melanocytes".

Down to a molecular level, Moncada et al. [56] found, using Raman spectroscopy and optical transmission spectroscopy, that in melasma lesions melanin is concentrated in the deeper layers of the skin (but its exteriorization is similar to that of healthy skin) and is sometimes structurally degraded. These data suggest a possible alternate explanation for hyperpigmentation and could also justify, at least in part, the variable efficacy of treatment protocols.

\section{Conclusions}

Often neglected by researchers and many clinicians because of its reputation of scientifically banal and purely cosmetic problem, melasma is recently being reconsidered thanks to an increased attention to the psychoemotional, other than physical, well-being of patients, and is showing its intrinsic and somehow fascinating complexity, and unexpected connections and interactions with several 
metabolic pathways. Further research efforts on this topic are strongly advisable, not only to improve current treatments, which are too often not effective as desired, but also -and maybe more important to gain a more complete understanding of the "melanin unit" and the physiology and pathophysiology of melanogenesis, a knowledge which could be of great help in the vast field of dyspigmentation disorders.

\section{References}

1. Handel AC, Miot LD, Miot HA (2014) Melasma: a clinical and epidemiological review. An Bras Dermatol 89: 771-782.

2. Grimes PE (1995) Melasma. Etiologic and therapeutic considerations. Arch Dermatol 131: 1453-1457.

3. Sheth VM, Pandya AG (2011) Melasma: a comprehensive update: part I. J Am Acad Dermatol 65: 689-697.

4. Tamega Ade A, Miot LD, Bonfietti C, Gige TC, Marques ME, et al. (2013) Clinical patterns and epidemiological characteristics of facial melasma in Brazilian women. J Eur Acad Dermatol Venereol 27: 151-156.

5. Elling SV, Powell FC (1997) Physiological changes in the skin during pregnancy. Clin Dermatol 15: 35-43.

6. Wolf R, Wolf D, Tamir A, Politi Y (1991) Melasma: a mask of stress. Br J Dermatol 125: 192-193.

7. Wu IB, Lambert C, Lotti TM, Hercogová J, Sintim-Damoa A, et al. (2012) Melasma. G Ital Dermatol Venereol 147: 413-418.

8. Ortonne JP, Arellano I, Berneburg M, Cestari T, Chan H, et al. (2009) A global survey of the role of ultraviolet radiation and hormonal influences in the development of melasma. J Eur Acad Dermatol Venereol 23: 1254-1262.

9. Vázquez M, Maldonado H, Benmamán C, Sánchez JL (1988) Melasma in men. A clinical and histologic study. Int J Dermatol 27: 25-27.

10. Moin A, Jabery Z, Fallah N (2006) Prevalence and awareness of melasma during pregnancy. Int J Dermatol 45: 285-288.

11. Achar A, Rathi SK (2011) Melasma: a clinico-epidemiological study of 312 cases. Indian J Dermatol 56: 380-382.

12. Goh CL, Dlova CN (1999) A retrospective study on the clinical presentation and treatment outcome of melasma in a tertiary dermatological referral centre in Singapore. Singapore Med J 40: 455-458.

13. Dynoodt, Mestdagh P, Van Peer G, Vandesompele J, Goossens K, et al. (2013) Identification of miR-145 as a key regulator of the pigmentary process. J Invest Dermatol 133: 201-209.

14. Im S, Kim J, On WY, Kang WH (2002) Increased expression of alphamelanocyte-stimulating hormone in the lesional skin of melasma. Br J Dermatol 146: 165-167.

15. Mahmoud BH1, Ruvolo E, Hexsel CL, Liu Y, Owen MR, et al. (2010) Impact of long-wavelength UVA and visible light on melanocompetent skin. J Invest Dermatol 130: 2092-2097.

16. Mahmoud BH, Hexsel CL, Hamzavi IH, Lim HW (2008) Effects of visible light on the skin. Photochem Photobiol 84: 450-462.

17. Castanedo-Cazares JP, Hernandez-Blanco D, Carlos-Ortega B, FuentesAhumada C, Torres-Álvarez B (2014) Near-visible light and UV photoprotection in the treatment of melasma: a double-blind randomized trial. Photodermatol Photoimmunol Photomed 30: 35-42.

18. Jee S, Lee SY, Chiu HC, Chang CC, Chen TJ (1994) Effects of estrogen and estrogen receptor in normal human melanocytes. Biochem Biophys Res Commun 199: 1407-1412.

19. Lieberman R, Moy $L$ (2008) Estrogen receptor expression in melasma: results from facial skin of affected patients. J Drugs Dermatol 7: 463-465.

20. Maeda K, Naganuma M, Fukuda M, Matsunaga J, Tomita Y (1996) Effect of pituitary and ovarian hormones on human melanocytes in vitro. Pigment Cell Res 9: 204-212.

21. Hassan I, Kaur I, Sialy R, Dash RJ (1998) Hormonal milieu in the maintenance of melasma in fertile women. J Dermatol 25: 510-512.

22. Mahmood K, Nadeem M, Aman S, Hameed A, Kazmi AH (2011) Role of estrogen, progesterone and prolactin in the etiopathogenesis of melasma in females. J Pak Assoc Dermatol 21: 241-247.
23. Pérez M, Sánchez JL, Aguiló $F$ (1983) Endocrinologic profile of patients with idiopathic melasma. J Invest Dermatol 81: 543-545.

24. Costin GE, Hearing VJ (2007) Human skin pigmentation: melanocytes modulate skin color in response to stress. FASEB J 21: 976-994.

25. Scott MC, Suzuki I, Abdel-Malek ZA (2002) Regulation of the human melanocortin 1 receptor expression in epidermal melanocytes by paracrine and endocrine factors and by ultraviolet radiation. Pigment Cell Res 15: 433-439.

26. Im S, Lee ES, Kim W, On W, Kim J, et al. (2002) Donor specific response of estrogen and progesterone on cultured human melanocytes. J Korean Med Sci 17: 58-64.

27. Miot LD, Miot HA, Polettini J, Silva MG, Marques ME (2010) Morphologic changes and the expression of alpha-melanocyte stimulating hormone and melanocortin-1 receptor in melasma lesions: a comparative study. Am J Dermatopathol 32: 676-682.

28. Kim NH, Cheong KA, Lee TR, Lee AY (2012) PDZK1 upregulation in estrogenrelated hyperpigmentation in melasma. J Invest Dermatol 132: 2622-2631.

29. Kim NH, Lee $\mathrm{CH}$, Lee AY (2010) H19 RNA downregulation stimulated melanogenesis in melasma. Pigment Cell Melanoma Res 23: 84-92.

30. Jang YH, Lee JY, Kang HY, Lee ES, Kim YC (2010) Oestrogen and progesterone receptor expression in melasma: an immunohistochemical analysis. J Eur Acad Dermatol Venereol 24: 1312-1316.

31. Resnik S (1967) Melasma induced by oral contraceptive drugs. JAMA 199: 601-605.

32. Martin AG1, Leal-Khouri S (1992) Physiologic skin changes associated with pregnancy. Int J Dermatol 31: 375-378.

33. Sialy R, Hassan I, Kaur I, Dash RJ (2000) Melasma in men: a hormonal profile. J Dermatol 27: 64-65.

34. Lutfi RJ, Fridmanis M, Misiunas AL, Pafume O, Gonzalez EA, et al. (1985 Association of melasma with thyroid autoimmunity and other thyroidal abnormalities and their relationship to the origin of the melasma. $\mathrm{J}$ Clin Endocrinol Metab 61: 28-31.

35. Yazdanfar A, Hashemi B (2010) Association of melasma with thyroid autoimmunity: a case-control study. Iran J Dermatol 13: 51-53.

36. Sacre RC, Fernandes NC, Vaisman M, Tendrich M (1996) Melasma idiopático: avaliação das funções tireoidiana, prolactínica e gonadal feminina. An Bras Dermatol 71: 195-198.

37. Passeron T (2013) Melasma pathogenesis and influencing factors - an overview of the latest research. J Eur Acad Dermatol Venereol 27 Suppl 1: 5-6.

38. Kim EH, Kim YC, Lee ES, Kang HY (2007) The vascular characteristics of melasma. J Dermatol Sci 46: 111-116.

39. Jo HY, Kim CK, Suh IB, Ryu SW, Ha KS, et al. (2009) Co-localization of inducible nitric oxide synthase and phosphorylated Akt in the lesional skins of patients with melasma. J Dermatol 36: 10-16.

40. Seçkin HY, Kalkan G, Bas Y, Akbas A, Onder Y (2014) Oxidative stress status in patients with melasma. Cutan Ocul Toxicol 33: 212-217.

41. Kang HY, Hwang JS, Lee JY, Ahn JH, Kim JY, et al. (2006) The dermal stem cell factor and c-kit are overexpressed in melasma. Br J Dermatol 154: 1094-1099.

42. Grichnik JM, Burch JA, Burchette J, Shea CR (1998) The SCF/KIT pathway plays a critical role in the control of normal human melanocyte homeostasis. J Invest Dermatol 111: 233-238.

43. Costa JJ, Demetri GD, Harrist TJ, Dvorak AM, Hayes DF, et al. (1996) Recombinant human stem cell factor (kit ligand) promotes human mast cell and melanocyte hyperplasia and functional activation in vivo. J Exp Med 183: 2681-2686.

44. Jang YH, Sim JH, Kang HY, Kim YC, Lee ES (2012) The histopathological characteristics of male melasma: comparison with female melasma and lentigo. J Am Acad Dermatol 66: 642-649.

45. Duarte I, Campos Lage AC (2007) Frequency of dermatoses associated with cosmetics. Contact Dermatitis 56: 211-213.

46. Negishi K, Kushikata N, Tezuka Y, Takeuchi K, Miyamoto E, et al. (2004) Study of the incidence and nature of "very subtle epidermal melasma" in relation to intense pulsed light treatment. Dermatol Surg 30: 881-886.

47. Hexsel D, Lacerda DA, Cavalcante AS, Machado Filho CA, Kalil CL, et al (2014) Epidemiology of melasma in Brazilian patients: a multicenter study. Int J Dermatol 53: 440-444. 
48. Adalatkhah H, Sadeghi Bazargani H2 (2013) The association between melasma and postinï $\neg$,ammatory hyperpigmentation in acne patients. Iran Red Crescent Med J 15: 400-403.

49. Sanchez NP, Pathak MA, Sato S, Fitzpatrick TB, Sanchez JL, et al. (1981) Melasma: a clinical, light microscopic, ultrastructural, and immunofluorescence study. J Am Acad Dermatol 4: 698-710.

50. Bak H, Lee HJ, Chang SE, Choi JH, Kim MN, et al. (2009) Increased expression of nerve growth factor receptor and neural endopeptidase in the lesional skin of melasma. Dermatol Surg 35: 1244-1250.

51. Kang HY, Suzuki I, Lee DJ, Ha J, Reiniche P, et al. (2011) Transcriptional profiling shows altered expression of wnt pathway- and lipid metabolismrelated genes as well as melanogenesis-related genes in melasma. J Invest Dermatol 131: 1692-1700.

52. Lee DJ, Lee J, Ha J, Park KC, Ortonne JP, et al. (2012) Defective barrier function in melasma skin. J Eur Acad Dermatol Venereol 26: 1533-1537.
53. Hernández-Barrera R, Torres-Alvarez B, Castanedo-Cazares JP, Oros-Ovalle C, Moncada B (2008) Solar elastosis and presence of mast cells as key features in the pathogenesis of melasma. Clin Exp Dermatol 33: 305-308.

54. Torres-Álvarez B, Mesa-Garza IG, Castanedo-Cázares JP, Fuentes-Ahumada C, Oros-Ovalle C (2011) Histochemical and immunohistochemical study in melasma: evidence of damage in the basal membrane. Am J Dermatopatho 33: 291-295.

55. Brianezi G, Handel AC, Schmitt JV, Miot LD, Miot HA (2014) Changes in nuclear morphology and chromatin texture of basal keratinocytes in melasma. J Eur Acad Dermatol Venereol .

56. Moncada B, Sahagún-Sánchez LK, Torres-Alvarez B, Castanedo-Cázares JP, Martínez-Ramírez JD, et al. (2009) Molecular structure and concentration of melanin in the stratum corneum of patients with melasma. Photodermatol Photoimmunol Photomed 25: 159-160.
This article was originally published in a special issue, Melasma handled by Editor(s). Dr. Serena Gianfaldoni, University of Pisa, Italy 